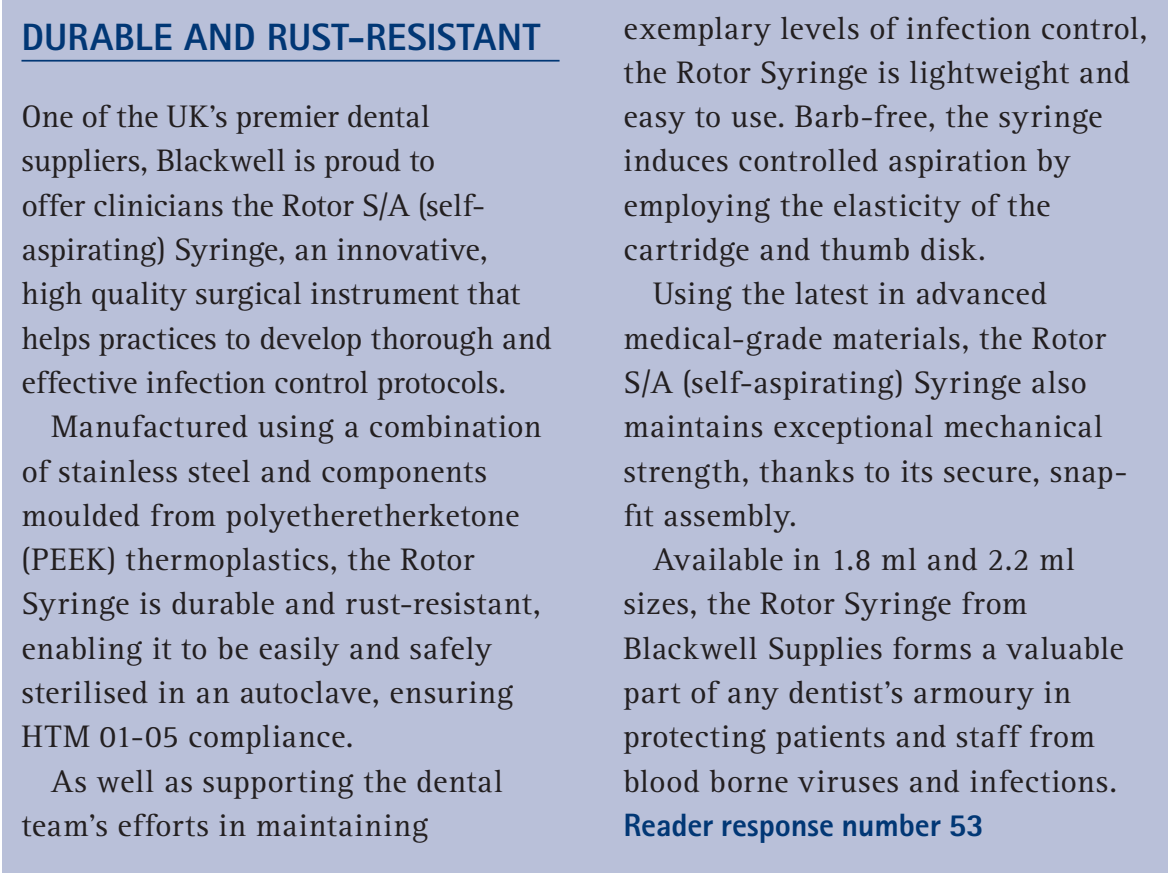

\section{NEW IMAGING BROCHURE}

The Dental Directory can help you plan, deliver and install your new imaging system with the minimum disruption to your surgery.

The Dental Directory's independent position means that they are not tied to offer you any particular system. The Dental Directory offers the most comprehensive range of digital imaging systems available from the world's leading manufacturers such as Schick, Gendex, Satelec, E-Woo and Durr.
The range of choice ensures that they can find the system that is right for you.

Attractive finance packages are also available from leading institutions, so whatever your requirements are for a new or upgrade system - The Dental Directory can help.

For the latest digital imaging systems available, The Dental Directory's Equipment and Digital Imaging Summer brochure is out now.

Reader response number 54

\section{CREATE QUALITY PROSTHETICS}

Nobel Biocare has been at the forefront of restorative dental solutions for over 40 years. Noble Biocare's experience has resulted in its latest technological achievement, NobelProcera. This advanced system provides the clinician with a quick and efficient method of creating a wide range of dental prosthetics including abutments, crowns, bridges, implant bridges and implant bar over denture varieties.

With its 3D prosthetic software and conoscopic holography, NobelProcera incorporates the latest in computer aided design and manufacture (CAD/CAM). This fully automated manufacturing process enables dental professionals to create sturdy and aesthetically pleasing prosthetics.

Furthermore, prosthetics need not be costly to the patient as NobelProcera allows a variety of materials to be used in the production process including titanium, alumina, acrylic and zirconia. This will allow greater flexibility when pricing your restorations and in turn many patients will no longer feel that restorative surgery is beyond their financial means, overall increasing your patient base.

The team at Nobel Biocare value their clients and are always available to give you advice on their products.

Reader response number 56

\title{
ECOFRIENDLY ELECTRONIC CIGARETTE
}

Nicolites are the most ecofriendly and advanced electronic cigarettes around. They contain no tobacco, tar, carbon monoxide or other harmful substances and can be smoked in all public places with no ash or cigarette ends but real smoking satisfaction.

Nicolite has no tar or carbon monoxide; nothing is lit or burnt and there is no tobacco smoke - instead a water vapour is exhaled that you can even blow smoke rings with. The odourless Nicolite tastes, feels and looks like the real thing.

Nicolite is reliable and consistent and comes ready to use. There is no charging necessary and one will last up to 25 traditional cigarettes' worth before needing to be disposed of. Traditional electronic cigarettes are fundamentally made up of three parts: a battery, an atomiser and a nicotine containing cartridge. With Nicolite the atomiser has been combined with the cartridge to make a more efficient, more reliable, simple to use product. Simply screw the two pieces together and Nicolite is ready to use.

Nicolite is also up to a third cheaper than traditional cigarettes (based on $£ 6.00$ for a pack of 20).

Reader response number 55 